\title{
Coastal flooding in Dieppe (Normandy) in a sea level rise context: numerical modelling, regulatory hazard mapping and simulations to reduce risk of marine submersion
}

\author{
Sylvestre LE ROY ${ }^{1}$, François PARIS ${ }^{2}$, Jérémy ROHMER ${ }^{1}$, \\ Rodrigo PEDREROS ${ }^{1}$, Sophie LECACHEUX ${ }^{1}$, Didier PENNEQUIN ${ }^{1}$, \\ Alexandre HERMENT ${ }^{3}$, Mélissa DELAVIE ${ }^{3}$, Luc MANGE ${ }^{4}$
}

\section{BRGM,}

3, avenue Claude Guillemin, BP 36009, 45060 Orléans Cedex 2, France.

s.leroy@brgm.fr

2. BRGM, désormais à Entente Oise-Aisne,

11 cours Guynemer, 60200 Compiègne, France.

3. Direction Départementale des Territoires et de la Mer de Seine-Maritime,

Cité administrative, 2, rue Saint-Sever - BP 76001, 76032 Rouen cedex, France.

4. Ville de Dieppe, Direction générale des Services techniques,

Centre technique municipal, 7, quai du Tonkin, 76200 Dieppe, France.

\section{Résumé :}

Dans le cadre de la révision du Plan de Prévention des Risques d'Inondation de la vallée de 1'Arques, la cartographie de l'aléa submersion marine sur Dieppe a été réalisée, à échéance actuelle et 100 ans, en suivant les prescriptions réglementaires nationales et en utilisant les outils les plus adaptés au contexte spécifique de la ville (débordement au niveau du port, franchissements en front de mer). Les résultats au niveau du centre-ville montrent que la ZAC Dieppe Sud est particulièrement exposée à la submersion.

En raison de projets de réhabilitation de la ZAC, il a alors été décidé d'étudier comment les aménagements prévus pourraient modifier cet aléa à l'échéance 100 ans. Cette analyse a montré que les aménagements prévus à court terme n'ont que des conséquences limitées sur l'aléa, mais que certaines mesures faciles à mettre en œuvre pouvaient permettre de limiter la submersion marine, et que les aménagements envisagés sur le long terme pourraient contribuer à encore la réduire.

\section{Mots-clés :}

Submersion marine, Modèle hydrodynamique, Cartographie d'aléa, Plan de Prévention des Risques, Changement climatique, Aménagement littoral.

\section{Introduction et contexte}

La ville de Dieppe se situe en Normandie, à l'embouchure de la vallée crayeuse de 1'Arques. Dans le cadre de la révision du Plan de Prévention des Risques d'Inondation de cette vallée, la Direction Départementale des territoires et de la Mer de Seine Maritime a chargé le BRGM de caractériser l'aléa "submersion marine" sur Dieppe et quelques 


\section{Thème 1 - Hydrodynamique côtière}

communes situées en amont. Ce travail achevé en 2017 a nécessité la construction d'une chaine de modélisation adaptée qui a non seulement permis d'établir la carte de l'aléa "submersion marine" sur Dieppe, mais qui a aussi été utilisée ensuite à des fins d'aménagement du territoire dans la ZAC Dieppe sud pour vérifier l'impact des futurs projets sur l'Aléa et pour trouver des solutions visant à en atténuer les effets. Le travail réalisé est décrit de manière succincte ci-après.

\section{Cartographie de l'aléa Submersion marine (LE ROY et al., 2017)}

\subsection{Cadre réglementaire}

La cartographie de l'aléa Submersion marine a été réalisée conformément au guide méthodologique national (MEDDE, 2014). Suivant les préconisations de ce guide, l'aléa a été étudié sur la base d'un événement de période de retour centennale au large en termes de niveau d'eau et de caractéristiques des vagues, en choisissant, selon les secteurs, le scénario le plus pénalisant en termes de volume d'eau franchissant.

Aucun ouvrage de protection contre les submersions marines n'a été identifié sur le secteur d'étude. Par contre, le fonctionnement des dispositifs hydrauliques (pertuis du port, clapets anti-retour au niveau de l'exutoire de la rivière) a été analysé de façon à prendre en compte les défaillances susceptibles de survenir et d'impacter la submersion marine sur le secteur.

L'aléa a été cartographié sur la base de simulations numériques à haute résolution, conduisant à la production de 2 cartes: une première carte, dite "Aléa actuel", correspondant au niveau marin actuellement observé augmenté de $0,2 \mathrm{~m}$, et une seconde carte, dite "Aléa à échéance 100 ans", correspondant au niveau marin actuellement observé augmenté de $0,6 \mathrm{~m}$ pour intégrer l'élévation du niveau de la mer sous l'effet du changement climatique.

\subsection{Définition des événements de référence}

Une analyse statistique aux probabilités conjointes des niveaux marins (mesurés à Dieppe par le marégraphe du SHOM) et des vagues au large a été réalisée pour identifier plusieurs combinaisons de période de retour centennale. Du fait de l'existence d'interactions marée-surcote dans cette partie de la Manche (IDIER et al., 2012 ; KERGADALLAN et al., 2016), cette analyse a été menée directement sur les niveaux mesurés, sans distinction spécifique des surcotes. L'analyse aux probabilités conjointes a permis de définir 13 combinaisons centennales (figure 1). En complément, il a été choisi d'imposer au niveau de la rivière un débit estimé comme étant le débit moyen journalier de période de retour annuelle. 


\section{XVI'̀mes Journées Nationales Génie Côtier - Génie Civil \\ Le Havre 2020}

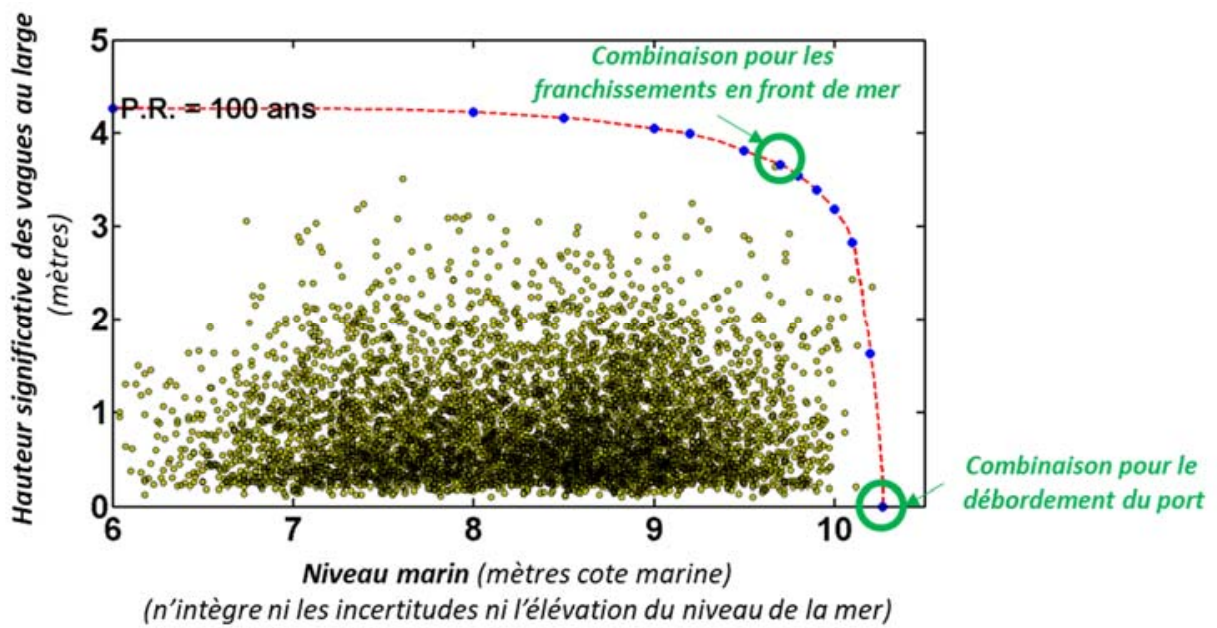

Figure 1. Courbe d'iso-probabilité de dépassement conjoint (niveau marin - vagues) centennale, combinaisons centennales étudiées et retenues selon les secteurs.

\subsection{Mise en place des modèles}

Une chaine de modélisation a été mise en place, nécessitant le traitement de diverses données pour des simulations réalistes (topo-bathymétrie, emprise des bâtiments, cartographie de coefficients de friction du sol sur les écoulements à partir de l'occupation du sol, ...). La chaine de modélisation s'appuie sur le modèle spectral WaveWatch III (propagation des vagues depuis le large jusqu'à la côte), le modèle de vagues SWASH (ZIJLEMA et al., 2011 ; simulation des franchissements de paquets de mer) et le modèle hydrodynamique MARS (LAZURE \& DUMAS, 2008 ; simulation des débordements et des écoulements de l'eau dans la zone inondée). Afin d'obtenir les résultats les plus réalistes possibles, l'inondation a été simulée à très haute résolution ( 2 mètres) au centreville de Dieppe, avec une prise en compte explicite du bâti dans le MNT pour éviter une sous-estimation des vitesses d'écoulement (LE ROY et al., 2015). Cette chaine de modélisation a été validée en reconstituant les débordements survenus au niveau du port pendant la tempête Xynthia.

Les combinaisons centennales issues de l'analyse aux probabilités conjointes se sont vues attribuer une chronologie sur 3 cycles de marée et ont été simulées de manière à identifier les situations les plus pénalisantes selon les secteurs (franchissements par paquets de mer au niveau des plages, débordement au niveau du port), afin de définir l'événement naturel de référence (cercles verts sur la figure 1). Les hypothèses de défaillance des ouvrages hydrauliques ont également été testées, ce qui a conduit à retenir pour la cartographie de l'aléa une hypothèse d'absence de ces ouvrages.

\subsection{Cartographie de l'aléa}

La cartographie de l'aléa a été réalisée sur la ville de Dieppe à partir de la simulation de l'événement naturel de référence. Les niveaux d'aléa ont été qualifiés par croisement 


\section{Thème 1 - Hydrodynamique côtière}

dynamique des hauteurs d'eau et des vitesses d'écoulement simultanés sur l'ensemble de la zone. Après un certain nombre de traitements spécifiques sous SIG (fusion des simulations, filtrages, intégration des bâtiments...), deux cartes ont ainsi été produites, correspondant respectivement à l'échéance actuelle et à l'échéance 100 ans, montrant que certains secteurs de la ville sont particulièrement exposés à la submersion marine, et notamment la ZAC Dieppe Sud. Ce sont ces cartes qui seront utilisées par les services de l'Etat pour réglementer l'urbanisation vis-à-vis de la submersion marine, figure 2.

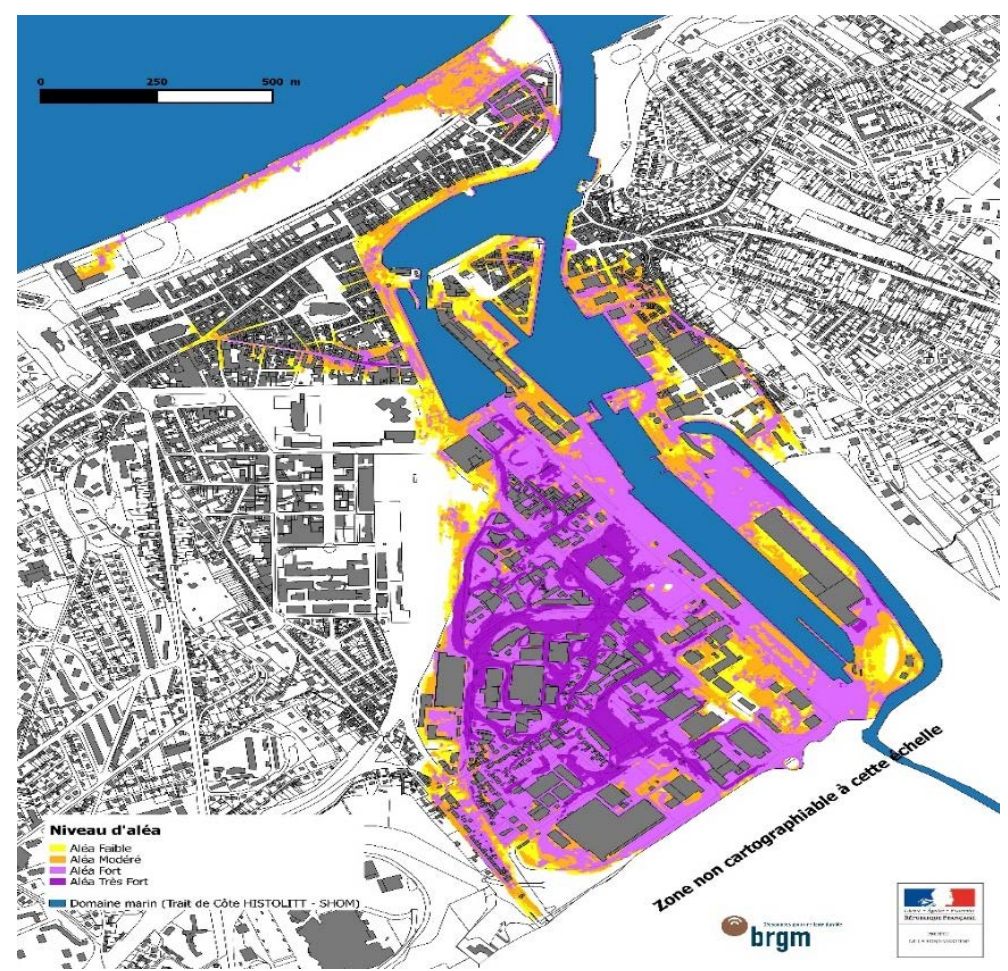

Figure 2. Aperçu de la carte d'aléa Submersion marine à échéance 100 ans au niveau du centre-ville de Dieppe et de la ZAC Dieppe Sud (échelle de validité 1/5 000̀me).

3. Scénarios d'aménagement et contrôle de la submersion marine (LE ROY, 2019) Compte-tenu des contraintes réglementaires apportées par le PPRI dans le secteur de la ZAC Dieppe Sud (où d'importants projets d'aménagements et de réhabilitation sont prévus), et du fait que la submersion marine sur ce secteur puisse être considérée comme prévisible et "progressive", la Ville de Dieppe et les services de l'Etat ont convenu de faire de la ZAC Dieppe-Sud une Zone à Réglementation Spécifique (ZRS), où des aménagements peuvent être envisagés sous réserve de s'assurer d'améliorer au mieux les conditions de la gestion de crise, de vérifier que la submersion n'est pas aggravée dans d'autres secteurs et de veiller à optimiser la sécurité des personnes et le retour à la normale après un événement. Par conséquent, la chaine de modélisation utilisée pour l'établissement des cartes d'aléa du PPRI a été exploitée ensuite pour visualiser l'impact des aménagements prévus et rechercher des moyens de contenir au mieux la submersion 


\section{XVİ̀mes Journées Nationales Génie Côtier - Génie Civil \\ Le Havre 2020}

et ses conséquences. Cette analyse a été réalisée en considérant le scénario à échéance 100 ans.

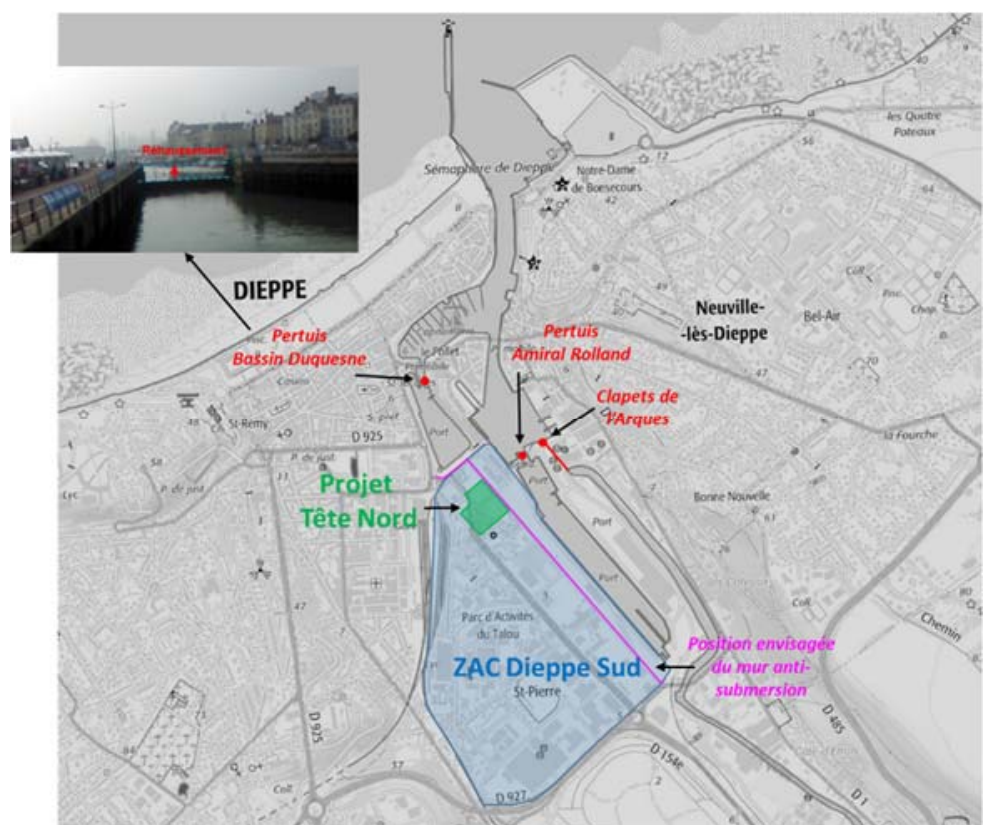

Figure 3. Localisation des dispositifs hydrauliques et des projets d'aménagements de la ZAC Dieppe Sud.

\subsection{Mesures envisagées}

Six mesures distinctes ont été envisagées et étudiées, dont 2 relèvent purement de l'aménagement du territoire (respectivement à court-terme et à long-terme), alors que les 4 autres correspondent à des aménagements visant à contrôler et limiter la submersion et ses conséquences. La localisation des mesures envisagées est présentée à la figure 3 .

- Mesure 1 : Aménagement prévu à court terme dans la Tête Nord de la ZAC ;

- Mesure 2 : Contrôle de la submersion par fermeture des 2 pertuis du port pendant la tempête (en fonctionnement normal actuel, les pertuis sont ouverts à marée haute et fermés à marée basse) ;

- Mesure 3 : Contrôle de la submersion par rehaussement du pertuis du bassin Duquesne jusqu'à la cote des quais (son sommet est actuellement 2,2 m sous la cote des quais); cette mesure n'a d'intérêt que combinée à la Mesure 2, dont elle amplifie l'effet atténuateur de la submersion ;

- Mesure 4 : Remblaiement de la Tête Nord de la ZAC pour mettre hors d'eau le projet à court-terme de la Mesure 1 ;

- Mesure 5 : Contrôle de la submersion par installation d'un dispositif de protection (type "mur anti-submersion") pour protéger la ZAC ;

- Mesure 6: Aménagement à long-terme: Projection à échéance 100 ans de l'urbanisation de la ZAC 


\section{Thème 1 - Hydrodynamique côtière}

\subsection{Définition des scénarios}

Les conséquences de ces 6 mesures sur la submersion dans la ZAC autour et ailleurs dans la ville, ont été simulées à travers 9 scénarios, établis sur la base de combinaisons de ces mesures, figure 4.

\begin{tabular}{|c|c|c|c|c|c|c|}
\hline & Mesure 1 & Mesure 2 & Mesure 3 & Mesure 4 & Mesure 5 & Mesure 6 \\
\hline & $\begin{array}{c}\text { Intégration des } \\
\text { aménagements } \\
\text { du projet " Tête } \\
\text { Nord " }\end{array}$ & $\begin{array}{c}\text { Adaptation du } \\
\text { mode de gestion } \\
\text { des } 2 \text { pertuis } \\
\text { (fermés durant la } \\
\text { tempête) }\end{array}$ & $\begin{array}{l}\text { Rehaussement du } \\
\text { pertuis du bassin } \\
\text { Duquesne à la } \\
\text { cote des quais }\end{array}$ & $\begin{array}{c}\text { Rehaussement } \\
\text { d'ensemble du } \\
\text { projet « Tête } \\
\text { Nord " }\end{array}$ & $\begin{array}{l}\text { Installation d'une } \\
\text { protection le long } \\
\text { des quais }\end{array}$ & $\begin{array}{l}\text { Intégration de } \\
\text { l'aménagement } \\
\text { prévisionnel du } \\
\text { reste de la ZAC }\end{array}$ \\
\hline Sc. 1 & $x$ & & & & & \\
\hline Sc. 2 & $\mathrm{X}$ & $\mathrm{X}$ & & & & \\
\hline Sc. 3 & $\mathrm{X}$ & $\mathrm{X}$ & $\mathrm{X}$ & & & \\
\hline Sc. 4 & $\mathrm{X}$ & & & $\mathrm{X}$ & & \\
\hline Sc. 5 & $\mathrm{X}$ & $\mathrm{X}$ & $\mathrm{X}$ & $x$ & & \\
\hline Sc. 6 & $x$ & & & $x$ & $\mathrm{X}$ & \\
\hline Sc. 7 & $x$ & & & $x$ & & $\mathrm{X}$ \\
\hline Sc. 8 & $x$ & $\mathrm{X}$ & & $\mathrm{X}$ & & $x$ \\
\hline Sc. 9 & $\mathrm{X}$ & $\mathrm{X}$ & $\mathrm{X}$ & $\mathrm{X}$ & & $\mathrm{X}$ \\
\hline
\end{tabular}

Figure 4. Combinaison des 6 mesures envisagées pour l'élaboration des 9 scénarios.

\subsection{Résultats obtenus}

Chaque scénario a ainsi été simulé à échéance 100 ans, et la submersion a été comparée à celles du PPRI en termes d'aléa (extension/réduction de l'inondation, modification des niveaux d'aléa) et de hauteurs d'eau, comme illustré pour le Scénario 3 à la figure 5.

La prise en compte du projet d'aménagement dans la Tête Nord de la ZAC (Mesure 1) ne conduit ainsi pas à des modifications substantielles de l'aléa. Après vérification auprès des architectes et urbanistes de la faisabilité d'un remblai permettant de mettre hors d'eau ce projet (Mesure 4), les simulations ont montré que cela n'induisait pas de modifications significatives de la submersion dans d'autres secteurs, bien que la zone inondée puisse s'en trouver légèrement plus étendue très localement.

Les mesures visant à contrôler la submersion marine dans la ZAC s'avèrent quant à elles très efficaces, à condition de s'assurer d'un dimensionnement suffisant des ouvrages impliqués face aux sollicitations auxquelles ils sont soumis : la fermeture des pertuis pendant la tempête (Mesure 2) retarde significativement le débordement du port, atténuant ainsi la submersion de manière importante. Cet effet peut encore être amplifié par le rehaussement du pertuis du bassin Duquesne jusqu'à la cote des quais (Mesure 3), retardant ainsi encore plus sa surverse, ce qui permet de réduire la hauteur d'eau dans la ZAC de 0,5 à 1,5 m, tout en épargnant substantiellement le centre-ville (figure 5). Si ces 2 mesures ne peuvent pas modifier le caractère inconstructible de certaines zones, elles peuvent toutefois constituer de bonnes mesures d'accompagnement pour le bâti existant. L'installation d'un dispositif anti-submersion pour protéger la ZAC permettrait d'épargner à cette dernière la majorité de l'inondation sans toutefois aggraver notablement la submersion sur les autres secteurs.

Enfin, l'aménagement prévisionnel de la ZAC à échéance 100 ans se traduit par une légère réduction des hauteurs d'eau et de l'aléa, notamment grâce à l'installation de bassins de rétention pouvant recueillir et stocker une partie de l'inondation. 


\section{XVİ̀mes Journées Nationales Génie Côtier - Génie Civil \\ Le Havre 2020}

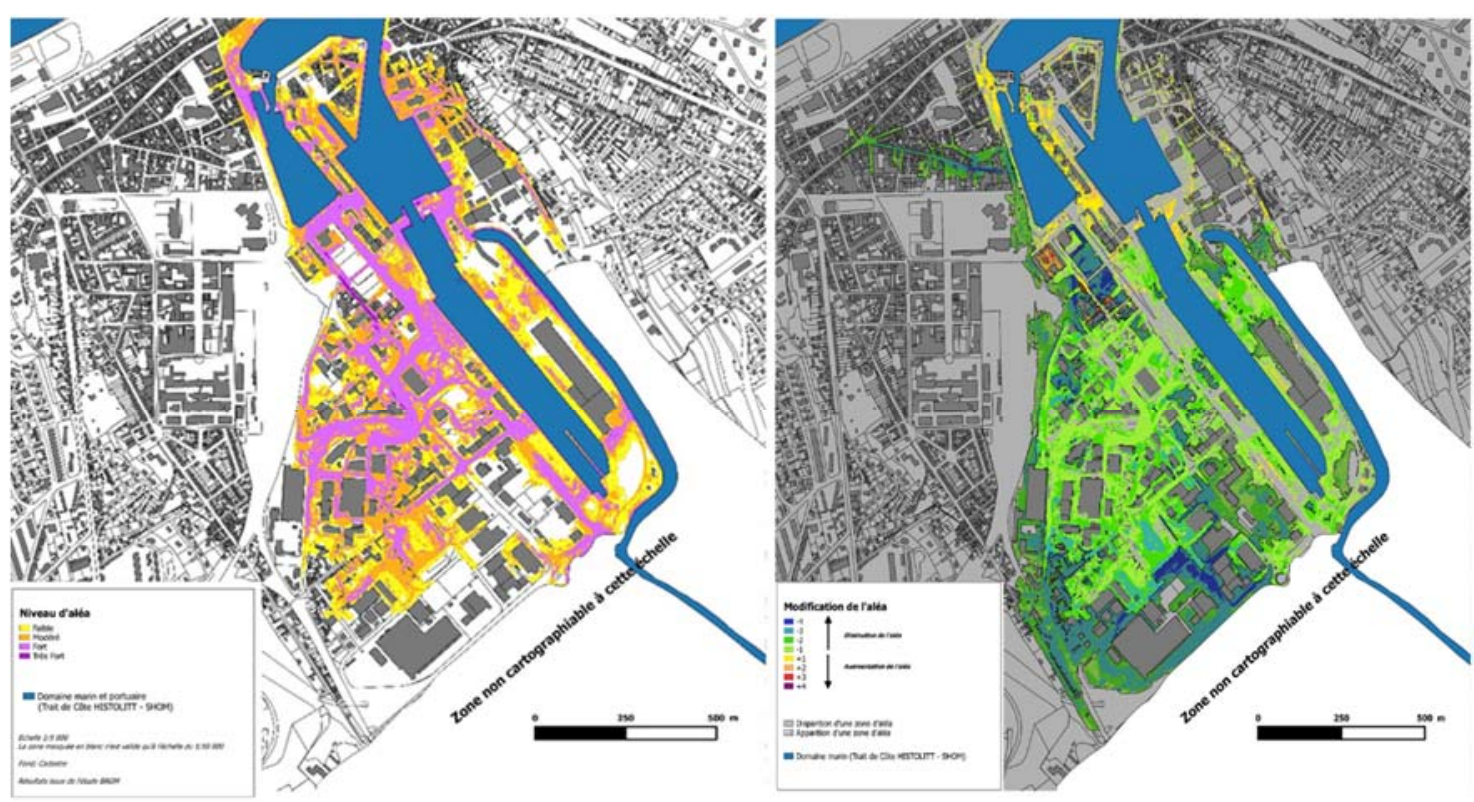

Figure 5. Carte d'aléa pour le Scénario 3 (Mesures 1, 2 \& 3) à gauche, et modification associée de la carte d'aléa par rapport à celle du PPRI à droite (jaune à rouge : augmentation de l'aléa ; vert à bleu : diminution de l'aléa ; en grisé : disparition de zones inondées).

\section{Conclusions}

Les nombreuses simulations réalisées sur la ville de Dieppe ont permis de quantifier les conséquences de l'élévation du niveau de la mer sur les submersions marines à Dieppe, bien que la ville soit à ce jour assez peu affectée par ce type de phénomènes. La ZAC Dieppe Sud, notamment, est particulièrement exposée, mais elle est aussi concernée par d'importants projets d'aménagement et de réhabilitation. Les simulations complémentaires ont permis de vérifier les conséquences des aménagements prévus sur l'inondation à échéance $100 \mathrm{ans}$, et de proposer des solutions qui permettraient de contrôler et limiter la submersion :

a) Le projet à court-terme dans la Tête Nord de la ZAC Dieppe Sud n'induit que peu de modifications de la submersion à échéance 100 ans ;

b) Ce projet peut être partiellement mis "hors d'eau" moyennant la création d'un remblai spécifique, mais cela peut conduire localement à une légère extension de la zone inondée sur d'autres secteurs ;

c) Une adaptation du fonctionnement des pertuis du port permettrait de limiter significativement la submersion autour du port ; Cet effet serait encore sensiblement amplifié en adaptant le pertuis du bassin Duquesne ;

d) L'installation d'un dispositif anti-submersion réduirait drastiquement le débordement dans la ZAC, sans amplifier significativement la submersion dans d'autres secteurs ; 


\section{Thème 1 - Hydrodynamique côtière}

e) L'aménagement prévisionnel du reste de la ZAC à échéance 100 ans tel qu'envisagé à ce jour se traduit par une légère réduction des hauteurs d'eau et de l'aléa, notamment du fait de l'implantation de bassins de rétention.

Ces résultats permettent désormais aux services de la ville et de l'Etat de réfléchir aux aménagements futurs dans la ZAC de Dieppe Sud et aux moyens de contrôler la submersion marine. Les cartes issues de ces scénarios d'aménagement ne se substituent toutefois pas à celles du PPRI, lesquelles doivent en tout état de cause prendre en compte des défaillances des divers ouvrages.

\section{Références bibliographiques}

IDIER D., DUMAS F., MULLER H. (2012). Tide-surge interaction in the English Channel. Nat. Hazards Earth Syst. Sci., Vol. 12, pp 3709-3718. https://doi.org/10.5194/nhess-12$\underline{3709-2012}$

KERGADALLAN X., BENOIT M., BERNARDARA P. (2016). Modélisation de la dépendance entre la marée et la surcote météorologique pour l'estimation des niveaux marins extrêmes en sites à fort marnage. Journées REFMAR, 02/04 Février 2016, Paris. LAZURE P., DUMAS F. (2008). An external-internal mode coupling for a 3D hydrodynamical model for applications at regional scale (MARS). Advances in Water Resources, Vol. 31, pp 233-250. https://doi.org/10.1016/j.advwatres.2007.06.010

LE ROY S. (2019). Evolutions de l'aléa "Submersion marine" à Dieppe selon des scénarios d'aménagement dans la ZAC Dieppe Sud. Rapport Final. BRGM/RP-68628FR, 117 p.

LE ROY S., PARIS F., ROHMER J., PEDREROS R., LECACHEUX S., RIDEL L., BULTEAU T., AYACHE B. (2017). Caractérisation de l'aléa submersion marine dans le cadre de la révision du PPRI de la Vallée de l'Arques - Cartographie de l'aléa sur les communes de Dieppe, Martin-Eglise et Rouxmesnil-Bouteilles. Rapport final. BRGM/RP-66697-FR, 133 p.

LE ROY S., PEDREROS R., ANDRE C., PARIS F., LECACHEUX S., MARCHE F., VINCHON C. (2015). Coastal flooding of urban areas by overtopping: dynamic modelling application to the Johanna storm (2008) in Gâvres (France). Nat. Hazards Earth Syst. Sci., Vol. 15, pp 2497-2510. https://doi.org/10.5194/nhess-15-2497-2015

MEDDE (2014). Guide Méthodologique, Plan de Prévention des Risques Littoraux. Version de mai 2014, 169 p.

ZIJLEMA M., STELLING G., SMIT P. (2011). SWASH: An operational public domain code for simulating wave fields and rapidly varied flows in coastal waters. Coastal Engineering, Vol. 58, pp 992-1012. https://doi.org/10.1016/j.coastaleng.2011.05.015 